\title{
Implementation and Comparison of Image Enhancement Techniques Using Low Resolution IRS-1C LISS III Image
}

\author{
Rubina Parveen ${ }^{1}$, Subhash Kulkarni ${ }^{2}$ and V. D. Mytri $^{3}$ \\ ${ }^{1}$ Research Scholar, VTU, Belagavi, Karnataka, India \\ ${ }^{2}$ PES Institute of Technology, Bangalore, Karnataka, India \\ Appa Institute of Engineering and Technology, Karnataka, India \\ E-Mail: rubina16det@gmail.com
}

\begin{abstract}
Image enhancement is the primary step in image processing. Image enhancement improves the interpretation and makes the image visually clear. In this process pixels of input image were fine-tuned, so that the results are more suitable for display or further image analysis. Numerical manipulation of digital image includes pre-processing as the preliminary step of analysis. Contrast manipulation, spatial filtering, noise suppression and color processing are different methods of image enhancement. Choosing suitable method for satellite image enhancement depends on the application. This paper aims to compare results of various image enhancement techniques using an IRS-1C LISS III satellite image. It attempts to assess enhancement techniques. Shortcomings and general requirements in enhancement techniques were also discussed. This study gives promising directions on research using IRS-1C LISS III image enhancement for future research. Keywords: Image enhancement, IRS-1C LISS III Image, Partial Differential Equations, Anisotropic Diffusion and Histogram Equalization
\end{abstract}

\section{INTRODUCTION}

Remote sensing images play a vital role for geo-spatial analysis. Visual appearance of the image can be improvised by better transform representation, for further automated image processing, such as classification, segmentation, analysis, detection and recognition [1]. Background information is important to understand satellite image features. IRS-1C LISS III (Indian Remote Sensing-1C Linear Self Scanning Sensor) images are low resolution images with $23.5 \mathrm{~m}$ resolution. Carrying out low level vision processing is a challenging task. Due to low resolution and mixed pixels, it is difficult to identify clearly and extract the land cover features. Enhancement techniques [2] have to be easily computed by reassignment of each pixel's gray level [3]. Initial processing of the raw data is done by removing noise and distortions. Enhanced image may not look like the input image [4]. Additional visual dimension is achieved by enhancement resulting in easier visual interpretation. Reducing noise in a satellite image is a challenging task for the application specific work [5]. Edge detection of the satellite image features significantly filters out redundant information, while preserving the important structural properties in an image. Edges are crucial to understand the shapes and boundaries of the image features [6]. Contrast stretching improves the visualization of objects in both darkest areas as well as lightest areas of the image at the same time [7]. In this paper, Contrast manipulation, Spatial filtering, Noise suppression and color processing image enhancement techniques were assessed on IRS-1C LISS III image. Filtering techniques handle color images corrupted by noise [8]. Area and filter window size are the two parameters provide direct control over which image features in case of selective enhancement [9]. Enhancement filter has to be adaptive to local changes in image statistics and should have the ability to smooth out noise [10], [11]. Anisotropic diffusion, Bilateral filter, Dark-frame subtraction, Fixed-pattern noise, Gaussian blur, Image noise reduction, Local pixel grouping, Median filter, Noise reduction, Non-local means, Salt-and-pepper noise removal, wiener de-convolution, Wiener filter are few of the techniques available in literature. Choosing any method for satellite image enhancement is application specific. Main objective of the paper is to implement various enhancement techniques using IRS-1C LISS III images and comparative assessment of resultant images visually.

\section{DATA SPECIFICATIONS}

The study image (shown in Fig. 1) is an IRS-1C LISS III data acquired on 02 nd march 2009 with a spatial resolution 23.5 sq. mts, provided by Karnataka State Remote Sensing Center, Regional Office Kalaburagi, Karnataka state, India.

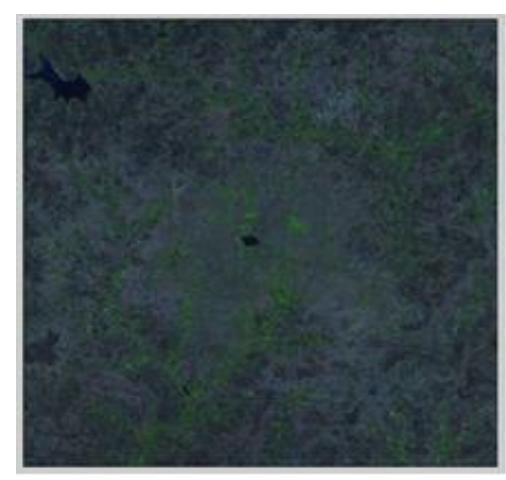

Fig. 1 Image Acquired on 02 March 2009

The Fig. 1 shows the area of analysis. LISS III multispectral image has four different spectral bands; it can be visualized as defining a 4-D space. 1st Band (0.45-0.52) is Blue band, 2nd Band (0.52-0.60) is Green band, 3rd Band (0.63-0.69) 
is Red band and 4th Band (0.76-0.90) is Short wave Infrared (SWIR) band. The study area is Kalaburagi District area, Karnataka State, India. It was acquired at 76.04' and 77.42 east longitude, and 17.12' and 17.46' north latitude. Image covers an area of $32.24 \mathrm{~km} / 3224.88$ hectares. Study area is predominantly urban city with very small water bodies and minimum vegetation. Simulation was performed by using MATLAB (R2010a) and ERDAS Imagine 9.1 software.

\section{ENHANCEMENT}

Objective of satellite image enhancement is to make an input image more suitable for further processing than original image, as par the requirements of specific application [12]. Improving the visual quality of images requires appropriate choice of enhancement technique, imaging modality, application at hand and viewing conditions. This study will provide a comparative analysis of image enhancement techniques. Following image enhancement methods were implemented and results were discussed.

\section{A. Decorrelation Stretch}

Color differences found LISS III multi-spectral data can be enhanced by removal of inter channel correlation found in pixels. Decorrelation stretch is a data visualization tool that shows as much discrimination of scene variations like vegetation types, geologic units, land uses/ land cover, etc. Decorrelation stretch enhances the color separation in multispectral image with high inter-channel correlation [13]; it can be quantitatively controlled based on the saturation levels of the image. Color ranges of the image were enhanced by preserving the relative sense of hue, saturation, and intensity. Enhanced image pixels will have a linear contrast stretch, with well distributed pixels among all possible colors [14]. The results of decorrelation Stretch applied on the study image were shown in Fig. 2 below.

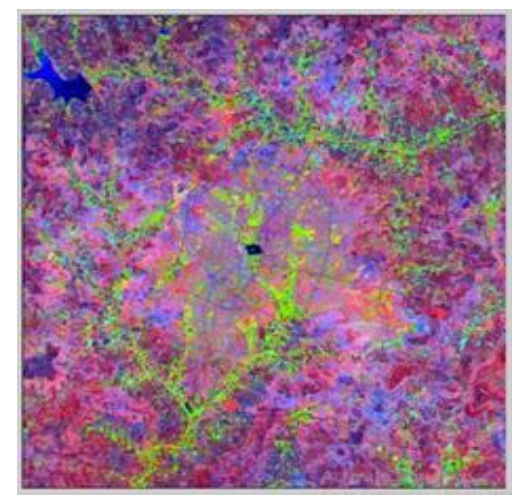

Fig. 2 Image Enhanced by Deccorelation Stretch

\section{B. Anisotropic Diffusion}

Anisotropic diffusion removes noise while preserving lowcontrast fine features and blurred thin edges by considering the gradient information of diffused pixels. Enhancement methods may inevitably take minute (fine) details as noise or vice versa. Fine details will have larger gray-level variance than the noisy background. This method effectively smooth's noise, simultaneously preserves edge and fine details [15].

Noise can be removed efficiently by keeping edges better at the same time to get good result on the whole image [16]. Let Input image frame $\mathrm{I}=(\mathrm{x}, \mathrm{y})$, denote the image intensity $(\mathrm{x}, \mathrm{y})$ which represents the pixel intensity values at $\mathrm{x}^{\text {th }}$ row and $y^{\text {th }}$ column. Gradients were given by $2 \mathrm{D}-$ gradient 5 $\mathrm{I}(\mathrm{x}, \mathrm{y})=\left(\mathrm{I}_{\mathrm{x}}, \mathrm{I}_{\mathrm{y}}\right)$. Diffusion coefficient $\mathrm{g}$ can be calculated by formula 1, with respect to time $t$. The results of anisotropic diffusion implemented on the study image are shown in Fig. 3.

$$
\frac{\partial I}{\partial t}=\nabla \cdot g(|\nabla I|) \nabla I
$$

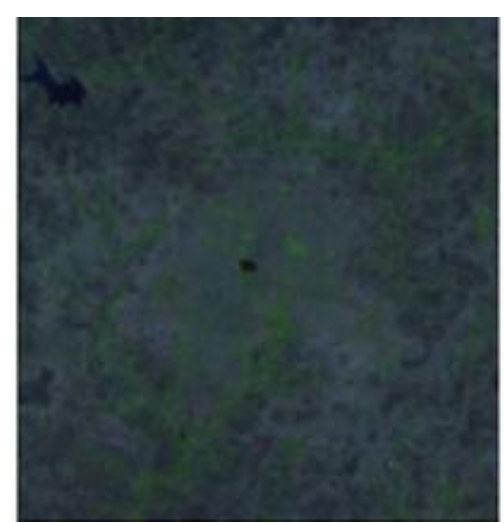

Fig. 3 Image Enhanced by Anisotropic Diffusion

\section{Partial Differential Equations}

Partial Differential Equations based method employees the similarity between the different band images in a multispectral image [17]. Noise free band of the image or noise free image was taken as a prior reference image, to develop a smoothing term. Smoothing teem refers to specific smoothing direction and a specific smoothing intensity of the reference image. Similarity of the directions of the edges between the noisy image and the reference image enables the PDE to smooth out noise and conserve detail in the denoising process. Fourth-order PDE model removes the noise on images which is signal dependent and difficult to remove [18], with superior performance on different Peak Signal Noise Ratio values and visual quality. $\delta x$ and $\delta y$ represents the partial derivatives of input image I with respect to $\mathrm{x}$ and $\mathrm{y}$. The gradient operator $\nabla \mathrm{I}$ is given by,

$$
\nabla I=\left(\frac{\partial I}{\partial x}, \frac{\partial I}{\partial y}\right)
$$

The resultant image of PDE based enhancement method was as shown in Fig. 4. 


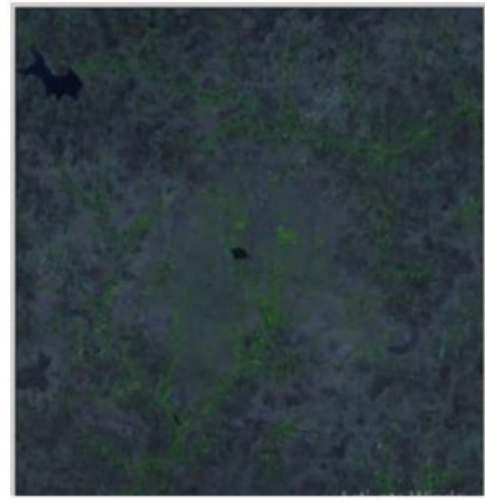

Fig. 4 Image Enhanced by PDE Method.

\section{Histogram Equalization}

Distortion caused by effect of atmosphere on radiations and instrumentation errors can be corrected by doing radiometric enhancement. Histogram Equalization (HE) is a useful technique for improving image contrast [19]. Unlike noise smoothing and contrast stretching methods, image is equalized for better visibility, using histogram equalization by changing the mean brightness of the multi-spectral image pixels [20].

This method performs better in preserving the brightness and producing more natural looking images with uniform histogram. HE is a simple and fast technique commonly used for image contrast enhancement [21]. Input image is normalized to range $[0,1]$ and let $\mathrm{p}(\mathrm{x})$ be the density function of intensity distribution of the input image, where $\mathrm{X}$ denotes the intensity value of the normalized image. Let $\mathrm{Y}$ be the output density function of intensity distribution of the HE image ( $\mathrm{Y}=1$ after equalization), by using the Equation 3.

$y=\int_{0}^{x} p(u) d u$

$\mathrm{u}$ is a dummy variable. Both $\mathrm{X}$ and $\mathrm{Y}$ are in $[0,1]$ range. The resultant HE image was shown in Fig. 5.
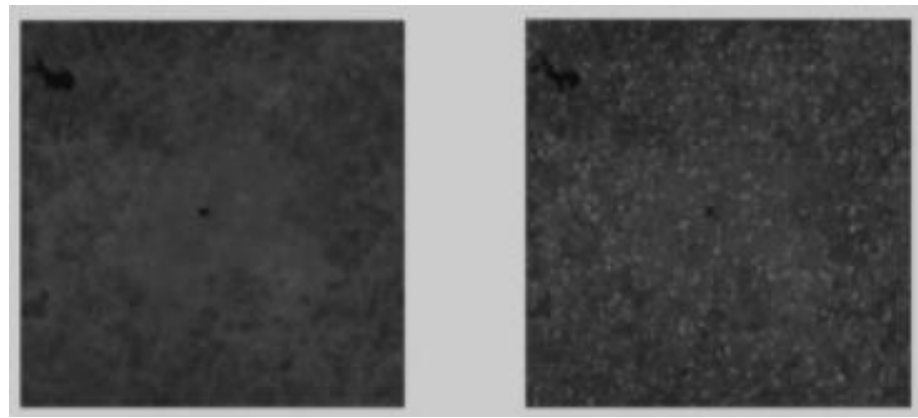

Fig. 5 Image Enhanced by Histogram equalization

\section{E. Enhancement by Noise and Haze reduction}

Earth rotation, panchromatic distortion, aspect ratio and sensor scan nonlinearities may cause Geometric errors. Spatial Enhancement method enhances an image using the values of individual and surrounding pixels. Radiometric Enhancement method enhances an image using the values of individual pixels within each band. Spectral Enhancement method enhances an image by transforming the values of each pixel on a multi-band basis. This can be done by various methods as discussed below.

1. Mean Filtering: Mean filtering replaces each image pixel value with the average value of its neighbors, including itself within defined kernel. Kernel represents the shape and size of the neighborhood to be sampled when calculating the mean. Some pepper and salt effect is sprayed on input image to make it little dirty. Applying the mean filter will result in little effect on the salt and pepper noise. This made noise blurred and smooth's image by reducing the amount of intensity variation between one pixel and the next. Mean filters reject noise and edges were better preserved than median filters [22]. Thus resulting in reducing noise in images. This effect is shown in Fig. 6 by converting color image into grey image. Multi-spectral image can be decomposed into RGB image, applying mean filtering on each RGB channel and concatenating after the filtering each channel, will give color mean filtering output.

Fig. 6 a. Input Image, b. Input Image with Added Noise and c. Mean Filtered Image

2. Median Filtering: Median filtering uses median values of pixels, including itself within defined kernel. It is a nonlinear operation often used in image processing to reduce "salt and pepper" noise. Median Filtering effect is 
shown in Fig. 7 by converting color image into grey image. Median filtering is less sensitive and it does not reduce the sharpness of the image. It can be observed that median filter removes noise better, with less blurring of edges.
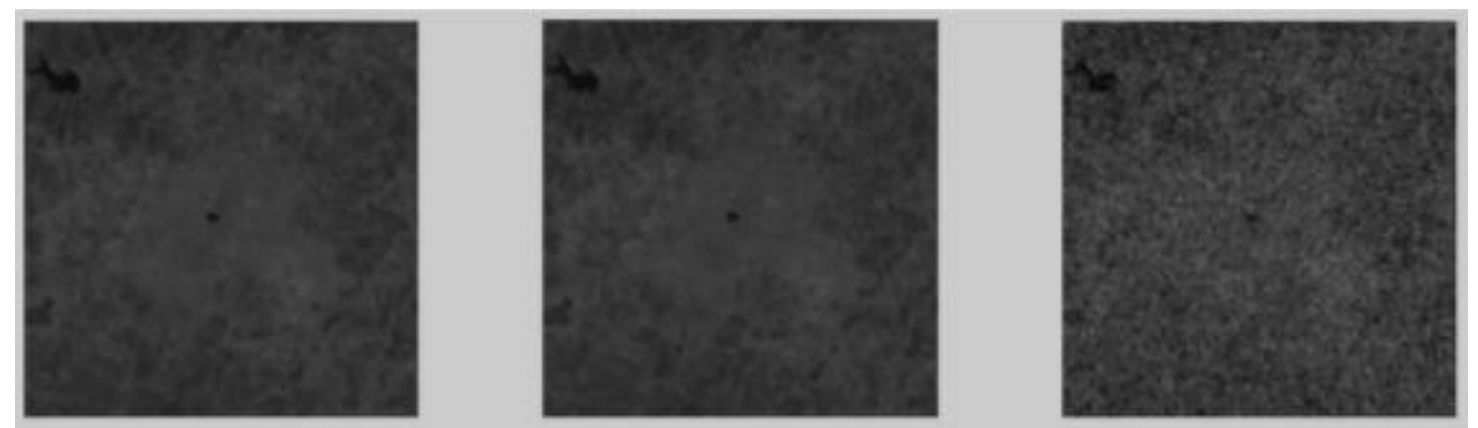

Fig. 7 a. Input image, b. Input image with added noise and c. Median filtered Image

3. Haze reduction: Atmospheric haze effects are like very fine particles, smoke, dust and liquid droplets suspended in the air limit the visibility and reduces contrast. Removing haze can significantly increase the visibility of the scene and correct the color shift [23]. Adaptive median filter identify pixels which are likely to be contaminated by noise [24] with edge preservation and noise suppression. Haze reduction enables to reduce overall haze in an input image. For LISS III images, Haze reduction based on the point spread convolution, generates a component that correlates with haze. This component is removed and the image is transformed back into RGB space. This method increases brightness, vegetation greenness, and surface moisture (wetness) as shown in Fig. 8. Bright areas like sand and barren land appear in red in color. Vegetation appears in green or cyan in color, fallow fields appear brownish in color and water bodies appear bright blue in color.

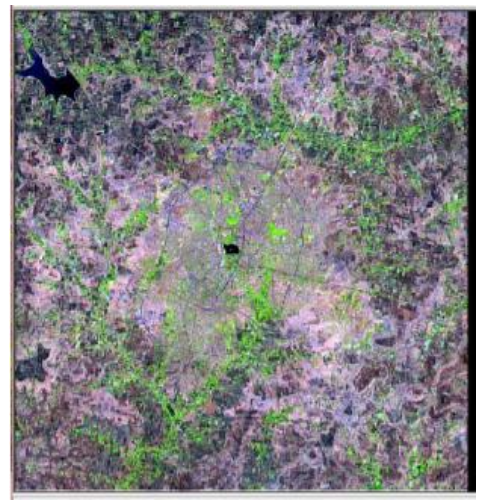

Fig. 8 Enhancement by Haze reduction

\section{F. Principal Component Analysis}

The Principal Component Analysis is a multispectral transformation method appeals directly to the vector nature of the multi-spectral image. It includes the principal component analysis transformation [25]. PCA reduces image dimensionality by defining new, four uncorrelated bands with orthogonal variables [26]. It provides maximum visual separability of image features thus maximum features appear in first principal component. It reduces the dimensionality by linear projection of data along the directions of maximal variance. Principal Component Analysis extracts the most important information from the image, compresses the size, simplify the description of the input image. Pixels of an image $\left(X_{1}, X_{2} . . X_{n}\right)$ given by $n-$ dimensional vector $\mathrm{x}=\left[\mathrm{X}_{1}, \mathrm{X}_{2}, \ldots \mathrm{X}_{\mathrm{n}}\right]^{\mathrm{T}}$. If the image $\mathrm{A}$ was of size $M^{*} N$ with four bands, Then, total $K=M * N$, 3-D vectors were generated. This was transformed into a vector $\mathrm{y}$ according to equation 4 .

$y=A\left(x-m_{x}\right)$

The vector $m x$ in equation 4 is the vector of mean values of all input variables. Correlation matrix, Eigen vectors and Eigen values were generated from the PCA. The Resultant first principal component image is shown in Fig. 9 below.

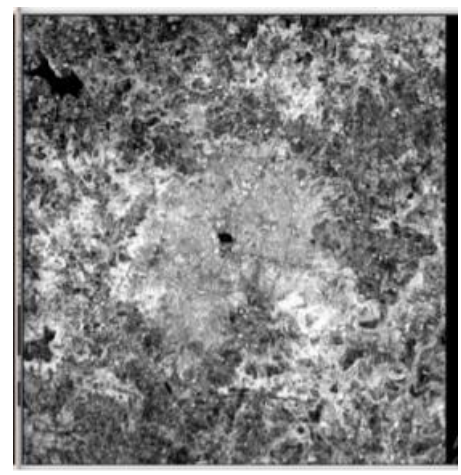

Fig. 9 Enhancement by Principal Component Analysis

\section{COMPARATIVE ANALYSIS}

Decorrection Stretch, shown in Fig. 2 reflects very bright colors. Excluding water and vegetation, but it was difficult to differentiate between the features. Anisotropic Diffusion [27] shown in Fig. 3 smooth's all the features, but it was difficult to determine the edges. Partial Differential Equation method as shown in Fig. 4 smooths out noise and conserves detail like edges. The output image is denoised and visually more appealing. Histogram Equalization as 
shown in Fig.5 develops darker shades of colors, but water and rocky lands were non-differentiable. Filtering methods like mean filters gives averaging effect suppresses the edges leading to blurring of an image, as shown in Fig 6 and Median Filter reduces the impact of salt and pepper noise with minimum blurring as shown in Fig 7. Haze reduction generates better output but gives blocky effect in the enhanced image as shown in Fig. 8. Principal Component Analysis method is an automatic and effective method to reduce the dimensionality but water and rocky lands are not much discriminable, as shown in .The first PC is the linear combination with maximal variance [28]. Compared to all the method implemented in the study, output generated by Partial Differential Method is visually better, removes the effect of noise, conserves edges and at the same time resultant image, as shown in Fig. 4 image feature images approximate the input image.

\section{CONCLUSION}

Enhancement is a preliminary step for any image processing technique to increase image quality needed as an input for further analysis. Enhancement results in improved visibility of the image by enhancing the contrast and simultaneous noise reduction. Main aim of the study was to make the multi-spectral image visually acceptable for display. LISS III images are low resolution images and differentiation of the feature at first look is difficult. A better enhancement technique is required to represent an input image in an appealing way. In this direction, various image enhancement techniques were tested on IRS-1C LISS III images. An assessment was made to find better enhancement techniques for LISS III images. Decorrelation stretch, Anisotropic Diffusion, Partial Differential Equation method, Histogram Equalization, Filtering and Principal Component Analysis were implemented on study image. It was observed visually, from the results obtained, that multispectral image enhancement by Partial Differential Equation overcomes the effect of noise and enables the user to identify the available features visually easily. However, the current work is still preliminary and yet promising. This study gives directions on research using low resolution multi-spectral IRS-1C LISS III image enhancement techniques for future research.

\section{REFERENCES}

[1] S. Bedi and R. Khandelwal, "Various Image Enhancement Techniques- A Critical Review", International Journal of Advanced Research in Computer and Communication Engineering, Vol. 2, No. 3, 2013.

[2] D. C. Wang, A. H. Vagnucci and C. Li, "Digital Image Enhancement: a Survey", Computer Vision, Graphics, and Image Processing, Vol. 24, No. 3, pp. 363-381, 1983.

[3] R. Hummel, "Image Enhancement by Histogram Transformation", Computer Graphics and Image Processing, Vol. 6, No. 2, pp. 184195, 1977.

[4] R. A. Schowengerdt, "Techniques For Image Processing And Classifications In Remote Sensing", Academic Press, 2012.

[5] M. V. Sarode and P. R. Deshmukh, "Reduction of Speckle Noise and Image Enhancement of Images using Filtering Technique", International Journal of Advancements in Technology, Vol. 11, 2011.
[6] R. Maini and H. Aggarwal, "Study and Comparison of Various Image Edge Detection Techniques", International Journal of Image Processing, Vol. 3, No. 1, pp. 1-11, 2009.

[7] S. S. Alamri, N. Kalyankar and S. Khamitkar, "Linear and NonLinear Contrast Enhancement Image", International Journal of Computer Science and Network Security, Vol. 10, No. 2, pp. 139$143,2010$.

[8] K. Tang, J. Astola and Y. Neuvo, "Nonlinear Multivariate Image Filtering Techniques", IEEE Transactions on Image Processing, Vol. 4, No. 6, pp. 788-798, 1995.

[9] C. Kenney, Y. Deng, B. Manjunath and G. Hewer, "Peer Group Image Enhancement", IEEE Transactions on Image Processing, Vol. 10, No. 2, pp. 326-334, 2001.

[10] D. T. Kuan, A. A. Sawchuk, T. C. Strand and P. Chavel, "Adaptive Noise Smoothing Filter for Images with Signal-Dependent Noise", IEEE Transactions on Pattern Analysis and Machine Intelligence, No. 2, pp. 165-177, 1985.

[11] R. Fattal, "Single Image Dehazing", ACM transactions on graphics (TOG), Vol. 27, No. 3, p. 72, 2008.

[12] W. Frei, "Image Enhancement by Histogram Hyperbolization", Computer Graphics and Image Processing, Vol. 6, No. 3, pp. 286294, 1977.

[13] R. Alley, "Algorithm Theoretical Basis Document for: Decorrelation Stretch", 1999.

[14] M. V. Dasu, V. Anitha, F. Shaik, and B. A. Rahim, "Feature Extraction of Satellite Images using Decorrelation Stretching and Color Scatter Plots", Digital Image Processing, Vol. 3, No. 14, pp. 873-877, 2011.

[15] S. M. Chao and D. M. Tsai, "An Improved Anisotropic Diffusion Model For Detail And Edge-Preserving Smoothing", Pattern Recognition Letters, Vol. 31, No. 13, pp. 2012-2023, 2010.

[16] M. H. Xie and Z. M. Wang, "Edge-directed Enhancing Based Anisotropic Diffusion Denoising", Dianzi Xuebao (Acta Electronica Sinica), Vol. 34, No. 1, pp. 59-64, 2006.

[17] P. Liu, F. Huang, G. Li and Z. Liu, "Remote-Sensing Image Denoising using Partial Differential Equations and Auxiliary Images as Priors", IEEE Geoscience and Remote Sensing Letters, Vol. 9, No. 3, pp. 358-362, 2012.

[18] B. Chen, J. L. Cai, W. S. Chen, and Y. Li, "A Multiplicative Noise Removal Approach Based on Partial Differential Equation Model", Mathematical Problems in Engineering, 2012.

[19] J. A. Stark, "Adaptive Image Contrast Enhancement using Generalizations of Histogram Equalization", IEEE Transactions On Image Processing, Vol. 9, No. 5, pp. 889-896, 2000.

[20] J. Singhai and P. Rawat, "Image Enhancement Method for Underwater, Ground and Satellite Images Using Brightness Preserving Histogram Equalization with Maximum Entropy", in Conference on Computational Intelligence and Multimedia Applications, 2007, International Conference on, Vol. 3, pp. 507512, IEEE, 2007.

[21] D. Menotti, A. d. A. Araujo, G. L. Pappa, L. Najman and J. Facon, "Contrast Enhancement in Digital Imaging using Histogram Equalization", in VII Workshop of Theses and Dissertations on Computer Graphics and Image Processing (WTDCGPI), part of SIBGRAPI, pp. 10, 2008.

[22] I. Pitas and A. Venetsanopoulos, "Nonlinear Mean Filters in Image Processing", IEEE Transactions on Acoustics, Speech and Signal Processing, Vol. 34, No. 3, pp. 573-584, 1986.

[23] K. He, J. Sun and X. Tang, "Single image haze removal using dark channel prior", IEEE Transactions on Pattern Analysis and Machine Intelligence, Vol. 33, No. 12, pp. 2341-2353, 2011.

[24] R. H. Chan, C. W. Ho and M. Nikolova, "Salt-and-pepper Noise Removal by Median Type Noise Detectors and Detail-Preserving Regularization”, IEEE Transactions on Image Processing, Vol. 14, No. 10, pp. 1479-1485, 2005.

[25] I. Jolliffe, Principal component analysis, Wiley Online Library, 2002.

[26] H. Abdi and L. J. Williams, "Principal Component Analysis", Wiley Interdisciplinary Reviews: Computational Statistics, Vol. 2, No. 4, pp. 433- 459, 2010.

[27] J. Weickert, “Anisotropic Diffusion in Image Processing", Vol. 1. Teubner Stuttgart, 1998.

[28] A. C. Rencher, "Principal Component Analysis Methods of Multivariate Analysis”, 2nd Ed., pp. 380-407, 2002. 